\title{
Realistic Experimentation Environments for Intelligent and Distributed Management and Orchestration (MANO) in 5G and beyond
}

\author{
Nina Slamnik-Kriještorac*, Paola Soto-Arenas*, Miguel Camelo Botero*, Luca Cominardi ${ }^{\dagger}$, \\ Steven Latré*, Johann M. Marquez-Barja* \\ * University of Antwerp - imec, IDLab - Faculty of Applied Engineering, Belgium \\ $\dagger$ ADLINK Technology, France
}

\begin{abstract}
As manual Management and Orchestration (MANO) of services and resources might delay the execution of MANO operations and negatively impact the performance of 5G and beyond Vehicle-to-Everything (V2X) services, applying AI in MANO to enable automation and intelligence is an imperative. The Network Function Virtualization (NFV), Software Defined Networking (SDN), and Artificial Intelligence (AI), could all together mitigate those challenges, and enable true automation in MANO operations. Thus, in this demo paper we will showcase the use of real-life testbed environments (Smart Highway and Virtual Wall, Belgium) and the Proof-of-Concept that we build to conduct realistic experimentation and validation of intelligent and distributed MANO in a dynamic network such as a V2X system.

Index Terms-management and orchestration, NFV, AI, ML,
\end{abstract} zenoh, testbeds, experimentation

\section{INTRODUCTION AND Motivation}

The services in $5 \mathrm{G}$ and beyond systems are strongly heterogeneous and diversified, and usually impose complex and potentially conflicting demands. To be able to adequately and timely adapt to changes in service configuration, as well as in traffic demand, networks need to be flexible, adaptable, and programmable [1]. Such requirements can be supported by technologies, such as Software Defined Networking (SDN), Network Function Virtualization (NFV), and Artificial Intelligence (AI) [2], in particular Machine Learning (ML), to provide automation and intelligence to the inherently manual MANagement and Orchestration (MANO) of services, thereby coping with the network complexity.

Although the potential of integrating AI into MANO operations is being recognized and studied both in the standardization and research, there is a lack of realistic experimentation and testing, where the true impact of $\mathrm{AI} / \mathrm{ML}$ on the optimization of NFV MANO operations is evaluated. There is a need for generating real traces to enhance the training of those $\mathrm{AI} / \mathrm{ML}$ algorithms, which is mainly done with synthetic data from simulators, as it has been demonstrated that their performance highly depend on using such real traces to finetune the resulting model [3]. Thus, in this demo paper we showcase our ongoing work towards building and fully utilizing the potential of high-performance real-life testbeds, such as Smart Highway ${ }^{1}$ [4] and Virtual Wall ${ }^{2}$, to enable testing and validation of intelligent and distributed NFV MANO in a dynamic network such as Vehicle-to-Everything (V2X) system.

II. ARChitecture of AI-ENHANCED MANO System

The integration of AI/ML into the NFV MANO systems for $5 \mathrm{G}$ and beyond is expected to mitigate the challenges imposed

${ }^{1}$ Smart Highway: https://www.fed4fire.eu/testbeds/smart-highway/

${ }^{2}$ Virtual Wall: https://www.fed4fire.eu/testbeds/virtual-wall/

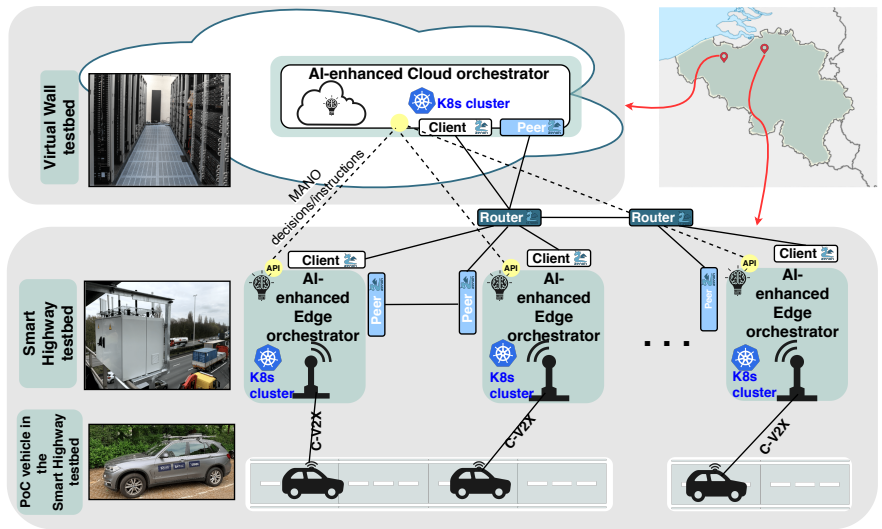

Fig. 1: The demo setup; Proof-of-Concept combining distributed testbed environments.

by manual orchestration (delayed deployment, scaling, service migration, etc.), as it is now mature enough to provide efficient solutions for complex optimization and prediction problems [1], such as optimized service instantiation, learning utilization patterns for computational resources of virtualized network services, proactive resource allocation/relocation, and optimized service migration.

Despite these benefits, AI/ML techniques need to be carefully chosen due the vulnerabilities in terms of security, scalability, high computation power (that might not be available in edge nodes), and the need for quality data to train the ML algorithms, as their performance on making decisions (e.g., predicting, classification, taking actions) will depend on how close was the training data to the actual data used in production environments.

To test and validate the impact of above mentioned challenges on the NFV MANO operations, we build and present a Proof-of-Concept (PoC) in real-life testbed environments, spanning users (vehicles), radio network (V2X), edge, and cloud infrastructure. The architecture of multi-domain MANO system presented in Fig. 1 is applicable to all distributed and heterogeneous softwarized networks whose operation stretches from edge to the cloud, where services and applications are usually deployed with microservice-based approach, and connectivity ensured via different wireless technologies including $5 \mathrm{G}$ and beyond [5].

As such networks are usually characterized by distributed resources belonging to different edge domains, which might belong to different Mobile Network Operators (MNOs), we follow the split between cloud (i.e., centralized) and edge 
orchestrators, which are deployed in a relationship $m: n$, $m<n, m, n \in \mathbf{N}$. The system enables autonomous MANO operations in each of the domains, but enforces an interplay between them for offloading orchestration decisions, or for retrieving data from distributed data engineering pipelines available in all edge domains.

\section{DEMO}

In Fig. 1, we map the testbed components to the elements of AI-enhanced MANO framework briefly presented in Section II. In this PoC, we provide the edge NFV infrastructure as distributed MEC hosts by virtualizing computational resources in Roadside Units (RSUs), which are deployed along the E313 highway in Antwerp, Belgium, as a part of the Smart Highway testbed. In each of these RSUs, we make use of the computational resources for performing lifecycle management of edge V2X services by deploying Kubernetes (K8s), where an AIenhanced edge orchestrator performs the role of K8s master and extends it to i) support cross domain operations, i.e., edgecloud interaction, and ii) receive dynamic triggers from AI/ML models deployed in Network Intelligent Functions (NIFs) for optimizing MANO operations. Such enhanced K8s master is in charge of deploying services and applications on the selected edge nodes. In this demo, both master and worker nodes will be deployed in Linux containers (LXC) on bare metal machines, as this proves to be a suitable practice for shared experimentation environments as testbeds.

As different types of data need to be collected to feed AI models (e.g., computational and network resource utilization, energy consumption, Key Performance Indicators (KPIs) measured at users' side, and users' locations), in this PoC we deploy Multi-Access Edge Computing (MEC) value-added services, as per definition in ETSI MEC [6], which perform data retrieval and pre-processing before publishing them on Zenoh [7]. Given its minimal network overhead (as little as $5 \mathrm{~B}$ ), and its small footprint (around $60 \mathrm{kB}$ on Arduino board), Zenoh is adopted in our PoC as a framework for data engineering pipeline. In particular, Zenoh provides a minimal set of primitives to deal with data in motion (e.g., real-time stream of vehicles' location/speed/destination), data at rest (e.g., historic data for vehicles' and edge nodes' computational resource utilization and energy consumption) and remote computations (e.g., on-demand calculation of the best route and speed limit). Each edge and cloud orchestrator acts as a subscriber for various types of data that can be stored on edges, and used for training or online learning/optimization.

Furthermore, concerning the vehicle as a client, our current PoC includes one vehicle that is capable to communicate with the edge services via long range $4 \mathrm{G}$ (to be extended to $5 \mathrm{G}$ in future). Thus, the client application is installed in the On-board Unit (OBU) of the vehicle, and it utilizes Uu link to exchange Cooperative Intelligent Transport System (C-ITS) messages with services, and inform them about its location, speed, heading, and destination. The testing service that we deploy on the edges for this demo is the backsituation awareness V2X service, which addresses emergency situations on the road, thereby proactively informing vehicles on the road about the arrival of an ambulance. This service is containerized and designed in a cloud-native way, and thus orchestrated by the edge orchestrator. Cloud orchestrator is running on the bare metal on top of the Virtual Wall testbed, located in Ghent, Belgium (Fig. 1). It is deployed as a web server (using Flask framework in python), which is capable of i) processing decision-offloading requests coming from the edge orchestrators, ii) location data processing and publishing on Zenoh, iii) injecting decisions on the northbound interface of edge orchestrators to instruct them to proactively migrate/relocate services from one edge to another, and iv) receiving notifications from NIFs deployed on the cloud, which enhance their operations and help them make efficient decisions on managing underlying resources and edge orchestrators. As a result, our demonstrator will show 1) the enhanced capabilities of the testbed to monitor and collect data, 2) the enhanced interfaces towards the orchestrators to consume and pre-process the data, and 3) an intelligent algorithm performing a MANO task to change the behavior of the system in an autonomous (closed-loop) way.

\section{ACKNOWLEDGEMENT}

This work has been performed in the framework of the European Union's Horizon 2020 project DAEMON co-funded by the EU under grant agreement No. 101017109, the Horizon 2020 Fed4FIRE+ project, Grant Agreement No. 723638, and the Horizon 2020 5G-Blueprint project under Agreement No. 952189.

\section{REFERENCES}

[1] D. M. Gutierrez-Estevez, M. Gramaglia, A. D. Domenico, G. Dandachi, S. Khatibi, D. Tsolkas, I. Balan, A. GarciaSaavedra, U. Elzur, and Y. Wang, "Artificial Intelligence for Elastic Management and Orchestration of $5 \mathrm{G}$ Networks," IEEE Wireless Communications, vol. 26, no. 5, pp. 134-141, 2019. doi: https://dx.doi.org/10.1109/MWC. 2019.1800498.

[2] ETSI, "Autonomous Networks, supporting tomorrow's ICT business," ETSI White Paper No. 40, 2020. Online [Available]: https://www.etsi.org/images/files/ ETSIWhitePapers/etsi-wp-40-Autonomous-networks.pdf.

[3] J. Tremblay, A. Prakash, D. Acuna, M. Brophy, V. Jampani, C. Anil, T. To, E. Cameracci, S. Boochoon, and S. Birchfield, "Training Deep Networks with Synthetic Data: Bridging the Reality Gap by Domain Randomization," in 2018 IEEE/CVF Conference on Computer Vision and Pattern Recognition Workshops (CVPRW), (Los Alamitos, CA, USA), pp. 1082-10828, IEEE Computer Society, jun 2018. doi: https://doi.org/10.1109/CVPRW. 2018.00143.

[4] J. Marquez-Barja, B. Lannoo, D. Naudts, B. Braem, V. Maglogiannis, C. Donato, S. Mercelis, R. Berkvens, P. Hellinckx, M. Weyn, et al., "Smart Highway: ITS-G5 and C2VX based testbed for vehicular communications in real environments enhanced by edge/cloud technologies," in EuCNC2019, the European Conference on Networks and Communications, pp. 1-2, 2019. Available [Online]:https://biblio.ugent.be/publication/8642435.

[5] N. Slamnik-Kriještorac, E. de Britto e Silva, E. Municio, H. Carvalho de Resende, S. Hadiwardoyo, and J. MarquezBarja, "Network Service and Resource Orchestration: A Feature and Performance Analysis within the MECEnhanced Vehicular Network Context," Sensors 2020, vol. 20, 2020. doi: https://doi.org/10.3390/s20143852.

[6] ETSI, "Multi-Access Edge Computing (MEC); Framework and Reference Architecture," ETSI ISG MEC, ETSI GS MEC 003 V2.1.1, 2019. Online [Available]: https://www.etsi.org/deliver/etsi_gs/MEC/001_099/ 003/02.01.01_60/gs_MEC003v020101p.pdf.

[7] Eclipse zenoh, "Project website: Eclipse zenoh," 2020. Online [Available]: https://zenoh.io, Last accessed on 2021-9-20. 